\title{
Tratamento da água Produzida Doce usando Processo Eletroquímico de Oxidação Avançada
}

\author{
Crislânia Carla de Oliveira Morais* (Mestranda do Programa de Pós-Graduação em Ciências Naturais - \\ PPGCN, na Universidade do Estado do Rio Grande do Norte - UERN); \\ André J ailson Cabral da Silva (Mestre do Programa de Pós-Graduação em Ciências Naturais - PPGCN, \\ na Universidade do Estado do Rio Grande do Norte - UERN); \\ Williane Simões Dantas (Técnica de laboratório de Química do Departamento de Química na \\ Universidade do Estado do Rio Grande do Norte - UERN); \\ Suely Souza Leal Castro (Prof. do Departamento de Química na Universidade do Estado do Rio Grande \\ do Norte - UERN). \\ * crislania morais@hotmail.com
}

\section{resumo:}

A indústria de petróleo lida com problemas de difícil solução em relação às atividades voltadas para a proteção ambiental, devido à geração de enormes quantidades de resíduos de alta complexidade e toxidez, como as águas de produção de petróleo. Atualmente, as técnicas de tratamento propostas (processos físicos e/ou biológicos) apresentam algumas limitações que dificultam o processo. Neste sentido, a oxidação eletroquímica surge como uma alternativa promissora para o tratamento de matrizes complexas como é o caso da água produzida. Esta técnica utiliza o elétron como reagente principal, tendo como vantagem uma alta eficiência na remoção da carga orgânica. Assim, esse trabalho teve por objetivo avaliar o desempenho do $\mathrm{ADE} \mathrm{Ti} / \mathrm{Ru}_{0,34} \mathrm{Ti}_{0,66} \mathrm{O}_{2}$ na degradação eletrolítica da Água Produzida Doce, independentemente de sua disposição final, reinjeção ou descarte. Foram avaliadas três densidades de corrente $\left(10,20\right.$ e $\left.30 \mathrm{~mA} \mathrm{~cm}^{-2}\right)$, a temperatura ambiente $\left(25 \pm 1^{\circ} \mathrm{C}\right)$. Para acompanhar o processo de degradação foi utilizada a demanda química de oxigênio (DQO). Os resultados mostraram que o processo de degradação é dependente da densidade de corrente aplicada e que o melhor desempenho foi obtido com a densidade de corrente de $20 \mathrm{~mA} \mathrm{~cm}^{-2}$, que removeu cerca de $96 \%$ de DQO com uma consumo de energia de $83,9 \mathrm{kWh} \mathrm{dm}^{-3}$. Apesar da densidade de corrente de $10 \mathrm{~mA} \mathrm{~cm}$ 2 ter tido um consumo de energia de $31,0 \mathrm{kWh} \mathrm{dm}^{-3}$, a remoção de DQO foi de apenas $71 \%$; já no caso da densidade de corrente de $30 \mathrm{~mA} \mathrm{~cm}{ }^{-2}$, a remoção de DQO foi $98 \%$, porém com um custo de 130 $\mathrm{kWh} \mathrm{dm}^{-3}$. Estes resultados mostraram que a utilização do ânodo $\mathrm{Ti} / \mathrm{Ru}_{0,34} \mathrm{Ti}_{0,66} \mathrm{O}_{2}$ pode ser uma alternativa de tratamento das águas de produção de petróleo.

pallavras-chave:

Efluentes petroquímicos; poluentes orgânicos; POA; eletro-oxidação.

Espaço reservado para organização do congresso. 


\section{I ntrodução}

Dos resíduos gerados pela indústria petroquímica, a água produzida (AP) é considerada como o principal agente poluidor devido ao grande volume, correspondendo a mais de $90 \%$ de todos os efluentes gerados na indústria do petróleo. Em média, para cada m³/dia de petróleo produzido são gerados de 3 a 4 m²/dia de água, podendo chegar a 7 ou mais nas atividades de exploração, perfuração e produção (RAMALHO MARTÍNEZ-HUITLE e SILVA, 2010; DIYA’UDDEEN et al., 2011; ROCHA et al., 2012).

A composição da AP é muito complexa, geralmente contém alto teor de sais, óleo disperso, metais dissolvidos, hidrocarbonetos, ácidos orgânicos, fenol, entre outros (RASHEED et al., 2011; ROCHA et al., 2012). Com isso, a sua disposição final tem um custo significativo, uma vez que as tecnologias utilizadas para o seu tratamento devem atender à legislação vigente (CONAMA 430/2011). $\mathrm{O}$ seu descarte ou reuso inadequado implicam em efeitos nocivos ao meio ambiente e à saúde da população.

Várias técnicas de tratamento de AP têm sido propostas na literatura. Entretanto, muitas apresentam limitações operacionais, tais como: geração de gases tóxicos, transferência de fase do poluente, produção de lodo residual, seletividade na oxidação de compostos refratários, demanda por grande área territorial, dentre outras limitações (DA SILVA et al., 2013).

Porém, nos últimos anos uma alternativa bastante promissora para o tratamento de efluentes de várias matrizes ambientais, inclusive os da indústria petrolífera, tem sido os processos eletroquímicos de oxidação avançada (PEOAs), que se baseiam na geração in situ de poderosos agentes oxidantes e não seletivos, conhecidos como radicais hidroxila $\left({ }^{\bullet} \mathrm{OH}\right), \mathrm{E}^{\circ}=2,8 \mathrm{~V}$ vs. $\mathrm{EPH}$, capazes de reagir com quase todas as classes de compostos, levando à formação de intermediários mais biodegradáveis ou à total mineralização, tendo como produtos finais do tratamento o $\mathrm{CO}_{2}$, a $\mathrm{H}_{2} \mathrm{O}$ e íons inorgânicos ( PANIZZA e CERISOLA, 2009; RESENDE et al., 2010; MARTÍNEZ-HUITLE e ROCHA, 2011; MORAIS et al., 2013).

Por utilizar semicondutores como catalizadores para a geração de radicais hidroxilas, através da oxidação de moléculas de água na sua superfície eletródica, os sistemas eletroquímicos são considerados tecnologias limpas em ascenção. Além de terem o elétron como reagente principal, apresentam algumas vantagens, como alta eficiência na remoção da carga orgânica, o uso de equipamentos simples e compactos, de fácil controle e manuseio (PANIZZA e CERISOLA, 2009, DA SILVA et al., 2013; MORAIS et al., 2013).

Os mecanismos de oxidação dos compostos orgânicos poderão ser influenciados não só por reações a partir da geração dos radicais hidroxilas (COMNINELLIS, 1994), mas também pela presença de outras espécies oxidantes contidas no efluente. Como as AP apresentam quantidades consideráveis de íons cloreto $\left(\mathrm{Cl}^{-}\right)$provenientes dos sais dissolvidos nesse efluente, durante as eletrólises estes íons podem ser convertidos a espécies de cloro $\left(\mathrm{Cl}_{2}\right)$, que poderão reagir rapidamente com a água formando o ácido hipocloroso e/ou hipoclorito dependendo do $\mathrm{pH}$ do meio (pKa 7,53), que por sua vez auxiliam no processo de degradação da matéria orgânica (REZENDE et al., 2010).

Vários estudos foram desenvolvidos usando os PEOAs para degradar substâncias em diversas matrizes, como efluentes têxteis (MARTÍNEZ-HUITLE e ROCHA 2011; MORAIS et al., 2013), fármacos (SIRÉS e BRILLAS, 2012), compostos fenólicos (GARG, MISHRA e CHAND, 2010; REZENDE et al., 2010), dentre outros.

Dentre os estudos sobre o tratamento eletroquímico de efluentes da indústria petroquímica, SANTOS et al. (2006), avaliaram a eficiência do anodo $\mathrm{Ti} / \mathrm{Ru}_{0,34} \mathrm{Ti}_{0,66} \mathrm{O}_{2}$ na eletro-oxidação de efluentes oleosos produzidos durante a extração e o refinamento de petróleo da planta da Petrobras/UNSEAL (Pilar, AL, Brasil). Os resultados mostraram que a remoção da carga orgânica foi dependente tanto da carga aplicada, quanto da temperatura e do tempo de eletrólise, sendo que a melhor condição foi obtida aplicando-se a densidade de corrente de $100 \mathrm{~mA} \mathrm{~cm}^{-2}$ à $50^{\circ} \mathrm{C}$, por um período de $70 \mathrm{~h}$.

RAMALHO, MARTÍNEZ-HUITLE E SILVA (2010), aplicaram tecnologia eletroquímica na remoção de hidrocarbonetos da água produzida gerada pela exploração do petróleo da planta da Petrobras/Plataforma de Curima, RN, Brasil, utilizando um reator de fluxo (Volume de 5 $\mathrm{dm}^{3}$ ) e um ADE de Ti/Ru0,23 $\mathrm{Ti}_{0,66} \mathrm{Sn}_{0,11} \mathrm{O}_{2}$. Os resultados indicaram aumento da eficiência de remoção dos compostos $(98,97,95$ e $84 \%)$ foi inversamente proporcianal à taxa de fluxo $(0,25$; 0,$5 ; 0,8$ e $\left.1,3 \mathrm{dm}^{3} \mathrm{~h}^{-1}\right)$. 
ROCHA et al. ( 2012), estudaram a oxidação eletroquímica da água produzida (planta da Petrobrás, RN, Brasil) sob condições galvanostáticas, utilizando ânodos de platina suportado em titânio (Ti/Pt) e diamante dopado com boro (DDB). Os resultados indicaram que o DDB foi o eletrodo que apresentou os melhores resultados, com uma remoção de $98 \%$ de DQO quando a densidade de corrente aplicada foi de $60 \mathrm{~mA} \mathrm{~cm}^{-2}$; quando o eletrodo de (Ti/Pt) foi utilizado nas mesma condições, a taxa de remoção de DQO foi de $80 \%$. O custo energético foi consideravelmente elevado $\left(139,52 \mathrm{KWh} \mathrm{dm}^{-3}\right.$ para o Ti/Pt e 109,80 KWh $\mathrm{dm}^{-3}$ para o DDB).

DA SILVA et al. (2013), avaliaram o tratamento eletroquímico da AP doce, salobra e salina geradas pela indústria petroquímica (planta da Petrobrás, RN, Brasil), usando $\mathrm{Ti} / \mathrm{IrO}_{2}-\mathrm{Ta}_{2} \mathrm{O}_{5}$ e DDB em reator de fluxo. Os resultados mostraram que no caso da AP doce a remoção completa ocorreu após 15 minutos de eletrólise na densidade de corrente de $5 \mathrm{~mA} \mathrm{~cm}{ }^{-2}$, usando o eletrodo de DDB à $25^{\circ} \mathrm{C}$; quando o ânodo Ti//rO $\mathrm{Ir}_{2}-\mathrm{Ta}_{2} \mathrm{O}_{5}$ foi usado, a remoção de DQO atingiu $90 \%$ em 15 minutos quando a temperatura foi elevada de $25^{\circ} \mathrm{C}$ para $40{ }^{\circ} \mathrm{C}$. No caso da AP salobra, a remoção completa de DQO foi obtida com o $\mathrm{Ti} / \mathrm{IrO}_{2}-\mathrm{Ta}_{2} \mathrm{O}_{5}$ na densidade de corrente de $30 \mathrm{~mA} \mathrm{~cm}{ }^{-2}$, após 240 minutos de eletrólise à $25^{\circ} \mathrm{C}$; e com o DDB a remoção completa foi obtida em todas as densidades de corrente, com um tempo muito menor. Quando à AP salina, foi obtido uma remoção de DQO de cerca de 50\%, para ambos os eletrodos e densidades de corrente aplicada (10 e $20 \mathrm{~mA} \mathrm{~cm}^{-2}$ ) .

Nesse trabalho, o objetivo foi avaliar o desempenho do $\mathrm{ADE} T \mathrm{Ti} / \mathrm{Ru}_{0,34} \mathrm{Ti}_{0,66} \mathrm{O}_{2}$ na degradação eletroquímica de efluentes reais da indústria de produção de petróleo (AP doce, salobra e salina), usando um sistema em batelada.

\section{MATERIAIS E MÉTODOS}

\subsection{Reagentes e amostras de água de produção}

Todos os experimentos foram realizados utilizando reagentes químicos de padrão analítico e água deionizada. A amostra da AP-Doce in natura foi fornecida pela Base da PETROBRAS, localizada no Estado do Rio Grande do Norte/Brasil. As características físico-químicas da amostra foram determinadas usando metodologias padrão (APHA, 2005), e encontram-se apresentadas na Tabela I. Com base na medida de salinidade, a AP foi classificada como doce.

\section{PARÂMETROS FÍSICO-QUÍMICOS AP-Doce}

\begin{tabular}{cc}
\hline $\mathrm{pH}$ & 7,7 \\
DQO $\left(\mathrm{mg} / \mathrm{L}\right.$ de $\left.\mathrm{O}_{2}\right)$ & 1.071 \\
Condutividade $(\mathrm{mS} / \mathrm{cm})$ & 0,6 \\
Salinidade $(\mathrm{mg} / \mathrm{L})$ & 40,0 \\
Cloreto $(\mathrm{mg} / \mathrm{L})$ & 24,3 \\
\hline
\end{tabular}

Tabela I: Resultados das análises físico-químicas das amostras de água produzida doce.

\subsection{Construção dos Eletrodos}

O ânodo foi construído a partir de telas de ADE de uso industrial, adquirido da De Nora do Brasil, em tela de $0,5 \mathrm{~mm}$ de espessura, com composição em mol de $34 \%$ de óxido de rutênio $\left(\mathrm{RuO}_{2}\right)$ e 66 \% de óxido de titânio $\left(\mathrm{TiO}_{2}\right)$, representado por $\mathrm{Ti} / \mathrm{Ru}_{0,34} \mathrm{Ti}_{0,66} \mathrm{O}_{2}$. As placas foram cortadas em formato retangular, com área geométrica de $13 \mathrm{~cm}^{2}$. Como cátodo foi utilizado duas placas de aço inox-316 totalizando uma área geométrica efetiva de $15 \mathrm{~cm}^{2}$. 


\subsection{Sistema Eletrolítico}

O tratamento eletroquímico foi conduzido em uma cela eletroquímica de compartimento único, contendo 250,0 mL da amostra de AP. Os eletrodos, cátodo e ânodo, foram montados em paralelo (ADE entre duas placas de aço inox), de forma que a distância entre eles se mantivesse em torno de 0,5 cm. As eletrólises foram efetuadas sob agitação constante, mantida com um agitador magnético da marca QUIMIS, e aplicação de corrente constante, por meio de uma fonte de alimentação da marca MINIPA, modelo MLP-3303, de 3A/5V (Figura I).

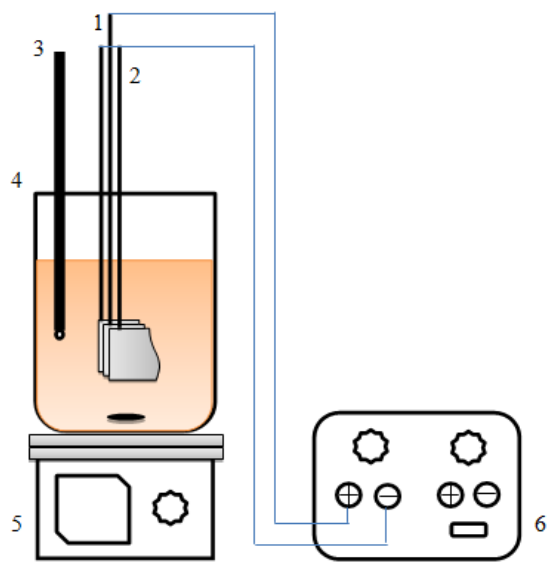

Figura I: Esquema do sistema eletroquímico, 1) ADE; 2) Cátodo; 3) Eletrodo de pH; 4) célula eletrolítica; 5) Agitador magnético e 6) fonte de alimentação.

Os experimentos de eletro-oxidação foram realizados à temperatura ambiente $\left(25 \pm 1{ }^{\circ} \mathrm{C}\right)$, nas densidades de corrente aplicada de 10, 20 e $30 \mathrm{~mA} \mathrm{~cm}$. $^{-2}$ Para acompanhar o processo de remoção da demanda química de oxigênio (DQO) durante as eletrólises, foram retiradas alíquotas de $2 \mathrm{~mL}$ a cada 30 minutos, por um período de 300 minutos. As análises foram feitas usando um fotômetro modelo HI 83099, da marca HANNA, após digestão das amostras na temperatura de $150{ }^{\circ} \mathrm{C}$ por um período de duas horas em um reator modelo HI 839800 e usando reagente faixa HI 93754B-25, ambos também da marca HANNA.

O potencial da célula foi medido a cada 30 minutos e os parâmetros físico-químicos, como pH e condutividade elétrica foram registrados antes e após as eletrólises, utilizando um medidor multiparamétrico modelo 5 Star, da marca Orion.

Para a limpeza dos eletrodos após cada eletrólise, foi aplicada uma corrente de 0,5 A durante cinco minutos em uma solução de $\mathrm{H}_{2} \mathrm{SO}_{4} 0,5 \mathrm{~mol} \mathrm{~L}^{-1}$ (PARRA et al., 2015)

\subsection{Consumo Energético}

O consumo energético (CE), em $\mathrm{kWh} \mathrm{dm}^{-3}$, que expressa a quantidade de energia consumida por unidade de volume de efluente tratado, foi determinado utilizando-se a Equação 1 (ROCHA et al., 2012).

$$
\mathrm{CE}=\frac{\Delta \mathrm{E}_{\mathrm{C}}}{3600 \mathrm{I}}
$$

Onde t é o tempo de eletrólise (s), $\Delta \mathrm{E}_{\mathrm{C}}$ é a média do potencial da célula (V), I a corrente de eletrólise (A) e V o volume da amostra $\left(\mathrm{dm}^{3}\right)$.

\section{Resultados e discussão}




\subsection{Processo de Degradação Eletroquímica}

A Figuras II, mostra o efeito da densidade de corrente aplicada (10, 20 e $\left.30 \mathrm{~mA} \mathrm{~cm}{ }^{-2}\right)$ sobre a remoção de DQO como uma função do tempo de eletrolise, usando o eletrodo de $\mathrm{Ti} / \mathrm{Ru}_{0,34} \mathrm{Ti}_{0,66} \mathrm{O}_{2}$, para as AP doce. A DQO foi utilizada para monitorar a evolução do processo de degradação durante as eletrólises, pois este parâmetro reflete a quantidade de matéria orgânica (biodegradável e refratária) removida durante o tratamento de efluentes.

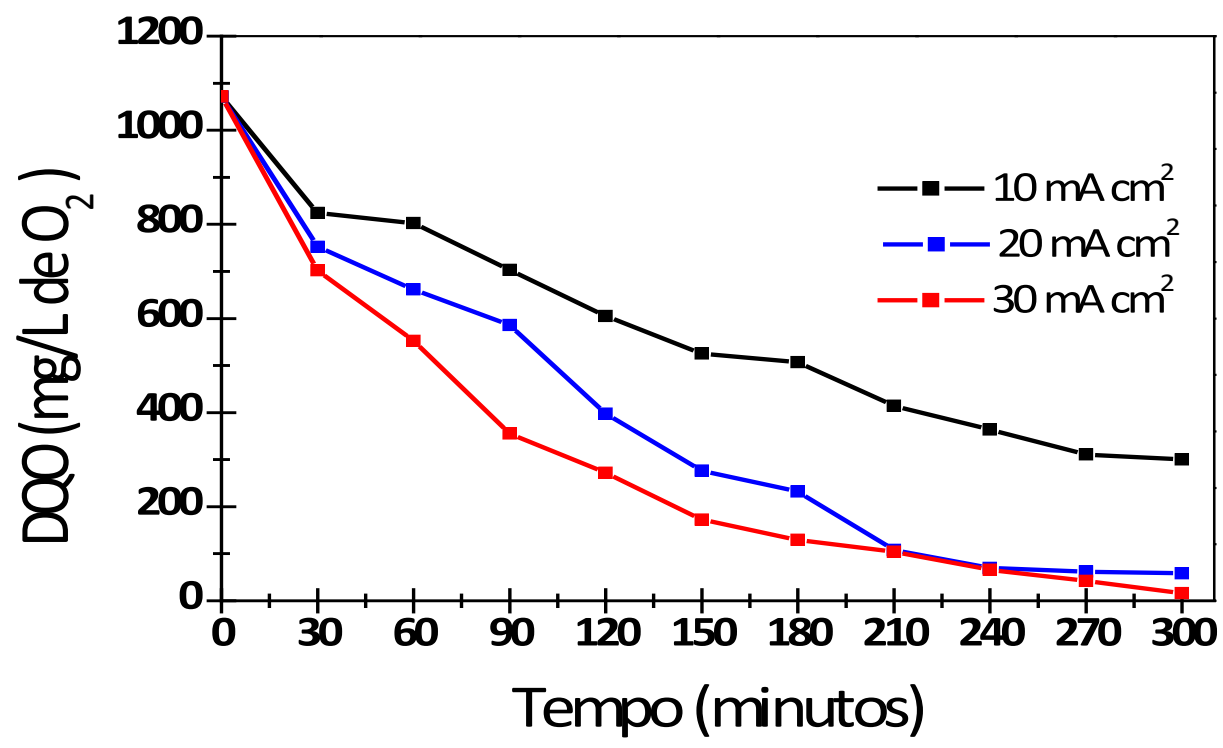

Figura II: Remoção da DQO em função do tempo de eletrólise durante o tratamento da AP-doce, em diferentes densidades de corrente aplicada.

De acordo com os resultados apresentados na Figura II, a velocidade da reação da degradação aumentou na medida em que a densidade de corrente aplicada foi aumentada de 10 para $20 \mathrm{~mA} \mathrm{~cm}{ }^{-2}$, com uma média de $96 \%$ remoção de DQO em 240 minutos na densidade de $20 \mathrm{~mA} \mathrm{~cm}{ }^{-2}$, enquanto que na densidade de $10 \mathrm{~mA} \mathrm{~cm}^{-2}$ a remoção chegou a $71 \%$ em 300 minutos de eletrólise. Este fato é devido à passagem de maiores cargas através da célula favorecendo uma maior geração de radicais ${ }^{\circ} \mathrm{OH}$, devido à eletrólise da água $\left(\mathrm{H}_{2} \mathrm{O} \rightarrow{ }^{\circ} \mathrm{OH}+\mathrm{H}^{+}+\mathrm{e}\right)$. Subsequentemente, esses radicais ${ }^{\circ} \mathrm{OH}$ interagem fortemente com vacâncias de oxigênio presentes na superfície do ânodo, formando os óxidos superiores $\left(\mathrm{MO}_{\mathrm{x}}\left({ }^{\circ} \mathrm{OH}\right)\right.$ $\rightarrow \mathrm{MO}_{\mathrm{x}+1}+\mathrm{H}^{+}+$e) que por sua vez oxidaram a matéria orgânica $\left(\mathrm{MO}_{\mathrm{x}+1}+\mathrm{R} \rightarrow \mathrm{MO}+\right.$ RO)(COMMINELLIS, 1994; ROCHA et al., 2012; DA SILVA et al., 2013).

Porém, quando a densidade de corrente foi aumentada de $20 \mathrm{~mA} \mathrm{~cm}{ }^{-2}$ para $30 \mathrm{~mA} \mathrm{~cm}$, observou-se que a remoção não foi tão significativa quando comparada com o aumento da densidade de corrente de $10 \mathrm{~mA} \mathrm{~cm}{ }^{-2}$ para $20 \mathrm{~mA} \mathrm{~cm}{ }^{-2}$. Esse resultado sugere que o $\mathrm{MO}_{\mathrm{x}+1}$ formado durante a eletrólise d'água favoreceu a reação da evolução de oxigênio molecular $\left(\mathrm{MO}_{\mathrm{x}+1} \rightarrow \mathrm{MOx}+1 / 2 \mathrm{O}_{2}\right)$ em detrimento da reação de oxidação da matéria orgânica. $\mathrm{O}$ aumento da produção de $\mathrm{O}_{2}$ na superfície anódica também pode ter desfavorecido o processo de adsorção dos compostos presentes na AP-Doce reduzindo assim a taxa de oxidação direta, uma vez que esta se baseia na transferência de elétrons entre a superfície do eletrodo e os substratos adsorvidos, como foram propostos por diversos autores para eletrodos tipo-ADE (TAVARES et al., 2012).

Adicionalmente, devido à variedade de compostos presentes no efluente, reações químicas secundárias podem ocorrer no meio reacional eletrolítico, tornando o processo de degradação muito complexo, uma vez que podem favorecer o processo ou, ao contrário, dificultar a degradação. Um exemplo é a presença de íons cloreto (Tabela I) que podem gerar espécies oxidantes $\mathrm{Como} \mathrm{Cl}_{2}, \mathrm{HOCl}$ e $\mathrm{OCl}^{-}$, que podem contribuir para a eletro-oxidação da matéria orgânica e, portanto, com a remoção da DQO; assim como também podem ser responsáveis pela formação de substâncias tóxicas, como os organoclorados (PANIZZA e CERISOLA 2009; RAMALHO et al., 2010; ROCHA et al., 2012; DA SILVA et al., 2013; SIRÉS et al., 2014). 
O mecanismo de eletrogeração de espécies de cloro ativo começa quando os íons cloretos são oxidados formando gás cloro, que por sua vez difunde-se, tanto em meio ácido como alcalino, formando grandes quantidades de ácido hipocloroso ou hipoclorito, conforme Equações 2 a 4, respectivamente ( SIRÉS et al., 2014).

$$
\begin{aligned}
& 2 \mathrm{Cl}^{-} \rightarrow \mathrm{Cl}_{2}+2 \mathrm{e}^{-} \\
& \mathrm{Cl}_{2}+\mathrm{H}_{2} \mathrm{O} \rightarrow \mathrm{HClO}+\mathrm{H}^{+}+\mathrm{Cl}^{-} \text {(meio ácido) } \\
& \mathrm{Cl}_{2}+2 \mathrm{OH}^{-} \leftrightarrow \mathrm{ClO}^{-}+\mathrm{Cl}^{-}+\mathrm{H}_{2} \mathrm{O} \text { (meio alcalino) }
\end{aligned}
$$

Analisando os pHs da AP-Doce, no início e no final das eletrólises, observa-se que este foi de 7,7 para cerca de 8,0 a 9,3 (Tabela II). Estes resultados são indicativos de que a espécie de cloro ativo predominante durante as eletrólises foi o íon $\mathrm{OCl}^{-}(\mathrm{E}=1,47 \mathrm{~V}$ vs. $\mathrm{EPH})$, apesar de que o $\mathrm{HOCl}(\mathrm{E}=$ $1,63 \mathrm{~V}$ vs. EPH) também podia estar ativo devido ao equilíbrio entre estas espécies nesta faixa de $\mathrm{pH}$ ) (SKOOG et al., 2006). Estes dados de potencial sugerem uma contribuição considerável das espécies de cloro ativo na remoção quase completa da DQO para a AP-Doce via oxidação indireta da matéria orgânica.

\begin{tabular}{ccccc}
\hline \multirow{2}{*}{$\begin{array}{c}\text { Água } \\
\text { produzida }\end{array}$} & $\mathbf{j}$ & \multicolumn{3}{c}{ Parâmetros avaliados } \\
\cline { 3 - 5 } & $\left(\mathbf{m A ~ \mathbf { ~ c m } ^ { - 2 } )}\right.$ & $\mathbf{p H}$ & $\begin{array}{c}\text { Condutividade } \\
(\mathbf{m S} \mathbf{~ c m})\end{array}$ & $\begin{array}{c}\Delta \mathbf{E}_{\mathbf{C}} \\
\mathbf{( V )}\end{array}$ \\
\hline \multirow{2}{*}{ Doce } & 10 & 8,0 & 0,313 & 11,8 \\
& 20 & 9,0 & 0,211 & 15,9 \\
& 30 & 9,3 & 0,132 & 16,3 \\
\hline
\end{tabular}

Tabela II: Parâmetros avaliados após o tratamento eletroquímico das amostras de água produzida doce, salobra e salina, em diferentes densidades de corrente (10, 20 e $\left.30 \mathrm{~mA} \mathrm{~cm}^{-2}\right)$.

\subsection{Estimativa do Consumo Energético}

Na Figura V encontram-se apresentados o consumo de energia para o tratamento da AP-Doce. Os resultados mostraram que os valores elevados podem ser atribuídos à baixa condutividade elétrica do meio reacional $\left(0,6 \mathrm{mS} \mathrm{cm}^{-1}\right)$, dificultando o fluxo de carga no meio eletrolítico, aumentando a resistencia hômica e, consequentemente, elevando o consumo de energia (RAMALHO et al., 2010; ROCHA et al., 2012; Da SILVA, 2013). Uma forma de reduzir esse custo seria a utilização de fontes alternativas de geração de energia, como por exemplo, a energia solar, ou aumentar a condutividade elétrica do meio reacional com a adição de íons, como por exempla o $\mathrm{NaCl}$ ou $\mathrm{Na}_{2} \mathrm{SO}_{4}$.

Levando em conta a relação remoção-custo, comparando-se as três densidades de corrente estudadas $\left(10,20\right.$ e $\left.30 \mathrm{~mA} \mathrm{~cm}{ }^{-2}\right)$, percebe-se que o melhor desempenho foi obtido com a densidade de corrente de $20 \mathrm{~mA} \mathrm{~cm}^{-2}$, que removeu cerca de 96\% de DQO com uma consumo de energia de 83,9 $\mathrm{kWh}$ $\mathrm{dm}^{-3}$. Apesar da densidade de $10 \mathrm{~mA} \mathrm{~cm}^{-2}$ ter tido um consumo de $31,0 \mathrm{kWh} \mathrm{dm}^{-3}$, a remoção de DQO foi de apenas 71,0 \%; já no caso da densidade de $30 \mathrm{~mA} \mathrm{~cm}^{-2}$ a remoção DQO foi de 98,0\%, porém o custo foi de $130 \mathrm{kWh} \mathrm{dm}^{-3}$. 


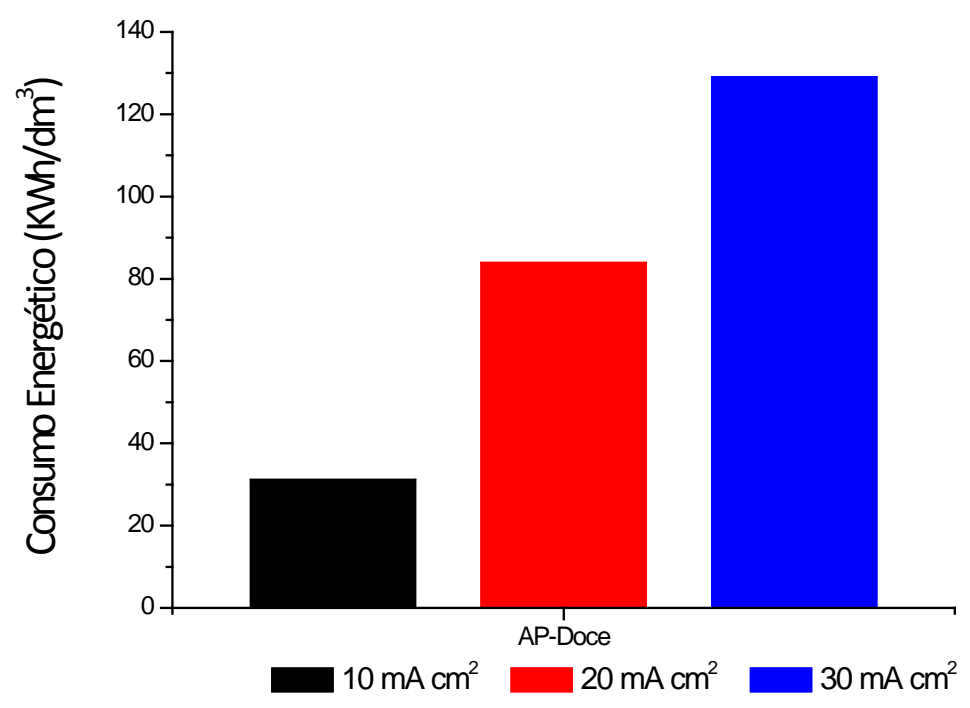

Figura V: Consumo energético para o tratamento eletroquímico da AP doce, salobra e salina, em diferentes densidades de corrente.

\section{Conclusão}

Os resultados mostraram que a utilização do ânodo $\mathrm{Ti} / \mathrm{Ru}_{0,34} \mathrm{Ti}_{0,66} \mathrm{O}_{2}$ pode ser uma alternativa promissora para o tratamento da AP-Doce, e que uma alternativa que pode ser usada para reduzir o custo do tratamento é a utilização de fontes renováveis de energia, ou a adição de íons no meio reacional.

\section{Produced sweet treat water using Electrochemical Advanced Oxidation Process}

Albstract: The oil industry deals with difficult problems in relation to activities aimed at environmental protection, due to the generation of huge quantities of waste high complexity and toxicity, as oil production waters. Currently, the proposed treatment techniques (physical and/or biological processes) have some limitations that hinder the process. In this regard, electrochemical oxidation arises as a promising alternative for the treatment of complex matrices such as produced water. This technique uses the electron as a primary reagent, the advantage of having a high efficiency in removing the organic load. Thus, this study aimed to evaluate the performance of $\mathrm{ADE} \mathrm{Ti} / \mathrm{Ru}_{0,34} \mathrm{Ti}_{0,66} \mathrm{O}_{2}$ the electrolytic degradation of Produced Water Sweet, regardless of its final disposition, reinjection or disposal. Were evaluated three current densities $\left(10,20\right.$ and $\left.30 \mathrm{~mA} \mathrm{~cm}{ }^{-2}\right)$ at room temperature $\left(25 \pm 1^{\circ} \mathrm{C}\right)$. To track the degradation process was used chemical oxygen demand (COD). The results showed that the degradation process is dependent on the applied current density and the better performance was obtained with current density of $20 \mathrm{~mA} \mathrm{~cm}^{-2}$, which removed about $96 \%$ of COD with a power consumption of $83,9 \mathrm{kWh} \mathrm{dm}^{-3}$. Although the current density of $10 \mathrm{~mA} \mathrm{~cm}{ }^{-2}$ have had a power consumption of $31.0 \mathrm{kWh} \mathrm{dm}^{-3}$, the COD removal was only $71 \%$; In the case of $30 \mathrm{~mA} \mathrm{~cm}^{-2}$ current density COD reduction was $98 \%$, but at a cost of $130 \mathrm{kWh} \mathrm{dm}^{-3}$. These results showed that use of the $\mathrm{Ti} / \mathrm{Ru}_{0,34} \mathrm{Ti}_{0,66} \mathrm{O}_{2}$ anode may be an alternative treatment in oil production water.

Keywords: Petrochemical effluents; organic pollutants; POA; electro-oxidation.

\section{Referências bibliográficas}


APHA, American Public Health Association. Standard Methods for the Examination of Water and Wastewater, Washington, 21th ed., 2005.

CERQUEIRA, A.A., MARQUES, M.R.C. RUSSO, C. Avaliação do Processo Eletrolítico em Corrente Alternada no Tratamento de Água de Produção. Química Nova, v. 34, n. 1, p. 59-63, nov. 2011.

COMNINELLIS, C.. Electrocatalysis in the electrochemical conversion/combustion of organic pollutants for waste water treatment. Electrochimica Acta, vol. 39, pp 1857-1862, 1994.

MMA - Ministério do Meio Ambiente. Conselho Nacional do Meio Ambiente. Resoluções do Conama: Resoluções vigentes publicadas entre setembro de 1984 e janeiro de 2012. Brasília: MMA, 2012. 1126 p. Acesso em: 15 de Setembro de 2015.

Disponível no site: http://www.mma.gov.br/port/conama/processos/61AA3835/LivroConama.pdf

DA SILVA, A.J.C., DOS SANTOS, E.V., MORAIS, C.C.O., MARTÍNEZ-HUILTLE, C.A.,CASTRO, S.S.L. Electrochemical treatment of fresh, brine and saline produced water generated by petrochemical industry using Ti/IrO2-Ta2O5 and BDD in flow reactor. Chemical Engineering Journal, v. 233, p. 47-55, Ago. 2013.

DIYA'UDDEN, B.H., DAUD, W.M.A.W., AZIZ, A.R.A. Treatment technologies for petroleum refinery effluents: a review. Process Safety and Environmental Protection, v. 89, n. 2 p. 95-105, Mar. 2011.

GARG, A.; MISHRA, I. M.; CHAND, S. Oxidative phenol degradation using non-noble metal based catalysts. Clean - Soil, Air, Water, v. 38, n. 1, p. 27-34, Jan. 2010.

MARTÍNEZ-HUITLE, C.A, ROCHA, J.H.B. Electrochemical technology for removing petroleum hydrocarbons from produced water. Exploration and Production: Oil and Gas Review, v. 9, n. 2, p. 110-116, 2011.

MORAIS, C.C.O.,DA SILVA, A.J.C., FERREIRA, M.B, ARAÚJO, D.M., ZANTA, C.L.P.S, CASTRO, S.S.L. Electrochemical Degradation of Methyl Red Using Ti/Ru0. 3Ti0. 7O2: Fragmentation of Azo Group. Electrocatalysis, v. 4, n. 4, p. 312-319, 2013.

PANIZZA, M., CERISOLA, G. Direct and Mediated Anodic Oxidation of Organic Pollutants. Chemical Review, v. 109, n. 12, p. 6541-6569, Dez. 2009.

PARRA, K. N. ; GUL, S. ; AQUINO, J. M. ; MIWA, D. W. ; MOTHEO, A. J. . Electrochemical degradation of tetracycline in artificial urine medium. Journal of Solid State Electrochemistry, v. 1, p. 1-9, Abr., 2015.

RAMALHO, A. M. Z., MARTÍNEZ-HUITLE, C. A., SILVA, D.R. Application of electrochemical technology for removing petroleum hydrocarbons from produced water using a DSA-type anode at different flow rates. Fuel, v. 89, n. 2, p. 531-534, Fev. 2010.

RASHEED, Q.J., PADIAN, K., MUTHUKUMAR, K. Treatment of petroleum refinery wastewater by ultrasound-dispersed nanoscale zero-valent iron particles. Ultrasonics Sonochemistry, v. 18, n. 5, p. 1138-1142, Set. 2011.

REZENDE, L. G. P., PRADO, V. M., ROCHA, R. S., BEATI, A. A. G. F., SOTOMAYOR, M. D. P., LANZA, M. R. V. Degradação eletroquímica do cloranfenicol em reator de refluxo. Quimíca Nova, v. 33, n. 5, p. 1088-1092, Abr. 2010. 
ROCHA, J.H.B., GOMES, M.M.S., FERNANDES, N.S., SILVA, D.R., MARTÍNEZ-HUITLE, C.A. Application of electrochemical oxidation as alternative treatment of produced water generated by Brazilian petrochemical industry. Fuel Processing Technology, v. 96, p. 80-87, Abr. 2012.

SANTOS, M.R.G., GOULART, M.O.F., TONHOLO, J., ZANTA, C.L.P.S. The application of electrochemical technology to the remediation of oily wastewater. Chemosphere, v. 64, n. 3, p. 393399, Jun. 2006.

SIRÉS, I., BRILLAS, E. Remediation of water pollution caused by pharmaceutical residues based on electrochemical separation and degradation technologies: a review. Environment International, v. 40, p. 212-219, Abr. 2012.

SKOOG, D. A.; WEST, D. M.; HOLLER, F. J.; CROUCH, S. R. Fundamento de Química Analítica, tradução da $8^{a}$ edição Norte americana, 2006, realizada por Marco Tadeu Grassi, revisada por Celio Pasquini, editora Cengage Learning, p. A-11, São Paulo, 2012.

TAVARES, M. G.; SILVA, L. V. A.; SOLANO, A. M. S.; TONHOLO, J.; MARTÍNEZ-HUITLE, C. A.; ZANTA, C. L. P. S.. Electrochemical oxidation of Methyl Red using Ti/Ru0,3Ti0,7O2 and Ti/Pt anodes. Chemical Engineering Journal, v. 204-206, p. 141-150, 2012. 